\title{
On determination of statistical properties of spectra from parametric level dynamics
}

\author{
Mirosław Hardej, ${ }^{1}$ Marek Kuś, ${ }^{1}$ Cezary Gonera, ${ }^{2}$ and Piotr Kosiński ${ }^{2}$ \\ ${ }^{1}$ Center for Theoretical Physics, Polish Academy of Sciences, \\ Aleja Lotników 32/44, 02-668 Warszawa, Poland \\ ${ }^{2}$ Department of Theoretical Physics II, University of Łódź, ul. Pomorska 149/153, 90-236 Eódź, Poland
}

\begin{abstract}
We analyze an approach aiming at determining statistical properties of spectra of time-periodic quantum chaotic system based on the parameter dynamics of their quasienergies. In particular we show that application of the methods of statistical physics, proposed previously in the literature, taking into account appropriate integrals of motion of the parametric dynamics is fully justified, even if the used integrals of motion do not determine the invariant manifold in a unique way. The indetermination of the manifold is removed by applying Dirac's theory of constrained Hamiltonian systems and imposing appropriate primary, first-class constraints and a gauge transformation generated by them in the standard way. The obtained results close the gap in the whole reasoning aiming at understanding statistical properties of spectra in terms of parametric dynamics.
\end{abstract}

PACS numbers: 05.45.Mt,05.45.a,05.40.-a

\section{INTRODUCTION}

One of the most characteristic features of quantum systems which exhibit chaotic behaviour in the classical limit is an affinity of their spectral properties to random matrices. The famous Bohigas-Giannoni-Schmidt conjecture [1] states that the statistics of distances between neighbouring energy levels of a quantum system with chaotic classical limit is well described by the one derived from the Random Matrix Theory (RMT) 2].

A vast numerical and experimental evidence [3, 4] in favour of this hypothesis was collected during last twenty years. There are also convincing theoretical arguments supporting it [5, 6]. In the present paper we would like to reconsider one of the first theoretical approaches initiated by Pechukas [7] and further developed by Yukawa [8, 9]. The original idea consisted in deriving differential equations describing parametric level dynamics i.e. the evolution of eigenvalues, when the parameter controlling the amount of chaos in the system changes, and applying the rules of classical equilibrium statistical mechanics to the flow described by the derived differential equations, treating the parameter as a fictitious time in which the "evolution" takes place. As observed by Yukawa the resulting dynamical system was Hamiltonian, hence applying rules of equilibrium statistical mechanics was straightforward; the equilibrium distribution should be given as the Boltzmann one,

$$
\rho=\mathcal{N} \exp (-\beta \mathcal{H})
$$

where $\mathcal{H}$ is the Hamilton function of the system, $\beta$ - a fictitious temperature (to be determined in some way from the initial data), and $\mathcal{N}$ - an appropriate normalization constant. The dynamical variables of the model, apart from the energy eigenvalues, involved also other ones. Integration over them over the phase space led to the equilibrium distribution of energy levels. As shown by Pechukas and Yukawa the resulting distribution coincides with those provided by RMT for the ensemble of real symmetric matrices with identically and independently distributed elements (forming the so-called Gaussian Orthogonal Ensemble).

As appealing and straightforward as the above outlined approach might be, one should not overlook some fundamental obstacles appearing when attempting to formulate it in a more rigourous way. Let us summarize briefly the most disturbing of them.

Both Pechukas and Yukawa started with the quantum Hamiltonian of the form

$$
H=H_{0}+\lambda V,
$$

where, according to their original interpretation, a time-independent $N \times N$ Hermitian matrix $H_{0}$ represented a quantum system enjoying integrable classical limit, whereas $V$ was an integrability-breaking part making the whole system classically fully chaotic when $\lambda$ attained appropriately large values. Thus the ambitious program was designed to actually investigate the transition between spectra of integrable $(\lambda=0)$ and nonintegrable $(\lambda$ - large $)$ cases. Thus, treating $\lambda$ as a fictitious time, one faces a problem belonging to non-equilibrium rather than equilibrium statistical mechanics, and usefulness of tools of the latter could be doubtful. Moreover, as it is clear from (2), as $\lambda$ grows the motion is unbounded, in particular the eigenvalues of $H$ grow indefinitely and, without an additional scaling, no 'equilibrium' distribution of eigenvalues of $H$ is attained (although it might be that the statistics of distances measured in units of the mean distance approaches some 'equilibrium'). There is a way of curing the situation - one 
should turn to dynamics of rescaled energy levels, what in fact consist in changing the $\lambda$-dependence of $H$ (see [3]). In the following we take another route - we would like to investigate the spectra of unitary evolution propagators instead of Hermitian Hamiltonians. Propagators being unitary have their spectra confined to the unit circle independently of their specific parameter dependence. The idea goes back to Dyson [10], although in slightly different context - he realized that it is more convenient to define probabilistic measures on ensembles of unitary rather than Hermitian matrices, what eventually led to the definitions of the circular ensembles of RMT.

In our case we do not pretend to mimic the same way. Instead we propose to start with the propagator for a particularly simple time-dependent quantum system in which the integrability breaking part in (2) has a form of periodic instantaneous kicks, so the whole Hamiltonian reads now:

$$
H(\lambda)=H_{0}+\lambda V \sum_{n=-\infty}^{\infty} \delta(t-n T),
$$

where $V$ is some constant, Hermitian matrix and $T$ the period of the perturbation.

The unitary evolution operator of the system (the propagator) is given as the solution of:

$$
i \hbar \frac{d F}{d t}=H(\lambda) F, \quad F(0)=I .
$$

Since the time dependence is periodic the whole information about the evolution of the system is encoded in $F$ evaluated at time $T$, i.e. the propagator transporting the system in time over one period of the perturbation. Since we will be concerned with properties of only this particular period-one propagator we will use $F$ to denote it without risking confusion with the general time-dependent one. In case it is needed we will write $F(\lambda)$ to remind its dependence of the perturbation parameter $\lambda$ inherited from the original Hamiltonian (31). In the following we put $\hbar=1$ and $T=1$.

In the case of a kicked system, the one-period evolution operator $F$ takes a particulary simple form

$$
F(\lambda)=\exp (-i \lambda V) F_{0}, \quad F_{0}:=\exp \left(-i H_{0}\right)
$$

Of our interest will be the eigenphases $\varphi_{n}(\lambda)$ (i.e. the phases of the eigenvalues), sometimes called also quasienergies of $F(\lambda)$,

$$
F(\lambda)\left|\phi_{n}(\lambda)\right\rangle=\exp \left(i \varphi_{n}(\lambda)\right)\left|\phi_{n}(\lambda)\right\rangle
$$

where $\left|\phi_{n}(\lambda)\right\rangle$ are eigenvectors of $F(\lambda)$.

Symmetries of the system in question (in particular with respect to the reversal of time) enforce additional symmetry conditions on $H$ and $F$. Without any other symmetries present, $H$ is a general hermitian and $F$ a general unitary $N \times N$ matrix (we will be mostly concerned with this case), whereas eg. for integer-spin time-reversal-symmetric systems $H$ is real symmetric $H^{*}=H=H^{T}$ and we can assume $F=F^{T}$ (i.e. $F$ symmetric).

As in the autonomous case (2) considered by Pechukas and Yukawa one can derive a closed set of differential equations, describing a Hamiltonian motion in a multi-dimensional phase-space with $\lambda$ as a fictitious time $([3,11$, , 12] $)$ (see next section). Since eigenvalues of an unitary matrix lie on the unit circle, their phases are restricted to an interval $[0,2 \pi[$, and in contrast to the autonomous case (2), the motion of the relevant variables is now bounded. This observation eases to some extend the above mentioned problems with the Pechukas-Yukawa approach, although does not warrant any equilibration as $\lambda$ grows. The problem of attaining the equilibrium was thoroughly discussed in [13. where it was shown that the time of effective equilibration goes to zero when approaching classical limit of the systems in question, hence the equilibrium statistical properties of the spectra should be detectable for large quantum numbers. We will recapitulate this discussion in the following sections.

When passing to the distribution of eigenphases from the equilibrium distribution (1) involving all dynamical variables one should choose an appropriate measure for integrating out the irrelevant variables. The natural measure is inherited from the symplectic structure underlying the parametric dynamics of the eigenphases and was discussed in [14.

Finally let us introduce briefly the main objective of the present paper aiming at closing the final gap in vindication of the parameter dynamics approach to statistical properties of quasi-energies outlined above. The postulated Boltzmann distribution can be validated only if there are no other constants of the motion apart from the Hamilton function itself, or in other words, when the motion is ergodic on the whole constant-energy surface. In the case of Pechukas-Yukawa parametric dynamics, as well as in the case of parametric motion of the kicked periodic systems (3) it is not the case. The dynamical systems governing the parametric motion of eigenvalues or eigenphases are so called generalized Calogero-Moser or Sutherland-Moser systems [15]. They posses many additional integrals of motion and in fact the 
motion is ergodic on a much smaller invariant manifold [16]. The simplest way to include the influence of additional integrals of motion consists of using in place of canonical ensemble measure (1) its grand-canonical generalization

$$
\rho \propto \exp \left\{-\sum_{\mu} \beta_{\mu} I_{\mu}\right\}
$$

nailing down the invariant manifold on which the motion takes place by fixing constants of the motion $I_{\mu}$ in the ensemble mean with the help of Lagrange parameters $\beta_{\mu}$; one of these $I_{\mu}$ should be the Hamilton function $\mathcal{H}$. Using the microcanonical ensemble $\rho \propto \prod_{\mu} \delta\left(I_{\mu}-\bar{I}_{\mu}\right)$ fixing the values of the constants of motion $I_{\mu}$ to their initial values $\bar{I}_{\mu}$ exactly, rather in the ensemble mean as (7) does, would be even more appropriate, but technically more complicated - see [3], Chapter 6 for a discussion of the problem.

Integration of $\rho$ over all dynamical variables except the eigenphases yields the desired distribution $P\left(\varphi_{1}, \ldots, \varphi_{N}\right)$. Such a program was performed in [17, 18] where it was shown that inclusion of additional known integrals of motion leads to corrections of the order $1 / N$ in comparison with the predictions of RMT. This result is highly satisfactory, since one expects convergence to RMT in the limit when the dimension of the matrix $N$ tends to infinity (which, in many models corresponds to classical limit of the quantum system). The only remaining problem is whether the integrals of motion taken into account in [17, 18] are all, which are needed to fix (in the ensemble mean) the invariant manifold on which the motion is ergodic, or in other words, what is the minimal set of independent integrals of motion determining the invariant manifold (see also [3] for the formulation of the problem). The independence of the integrals used in the above mentioned papers was investigated in [19]. In the present paper we close the last gap by showing that they determine the invariant manifold to the extent which is appropriate to infer the distribution of eigenphases.

In Section [1] we briefly derive the dynamical equations for the parametric motion of eigenphases, whereas in Section III we discuss the statistical mechanics of the one-dimensional gas of eigenphases. Section IV is devoted to additional integrals of motion: we give their form appropriate for further applications and briefly discuss their independence. The final Section $\nabla$ offers the ultimate solution of the problem in frames of the theory of constrained Hamiltonian systems.

\section{PARAMETRIC LEVEL DYNAMICS}

Let $W(\lambda)$ diagonalizes $F(\lambda)$,

$$
\begin{array}{r}
W(\lambda) F(\lambda) W^{-1}(\lambda)=e^{-i \Phi(\lambda)}=\operatorname{diag}\left(e^{-i \varphi_{1}(\lambda)}, \ldots, e^{-i \varphi_{N}(\lambda)}\right), \\
\Phi(\lambda)=\operatorname{diag}\left(\varphi_{1}(\lambda), \ldots, \varphi_{N}(\lambda)\right) .
\end{array}
$$

In the following we shall skip exhibiting the explicit $\lambda$-dependence when possible. Since $F$ is unitary, so is $W$, $W^{-1}=W^{\dagger}$. In the case of a general unitary matrix, the diagonalizing matrix $W$ is not unique even after ordering the eigenphases: we can always left-multiply it by a diagonal unitary matrix without altering the result (8).

Let's define following auxiliary matrices

$$
\begin{aligned}
v & :=W V W^{-1}=v^{\dagger}, \\
l & :=i e^{i \Phi}\left[v, e^{-i \Phi}\right]=-l^{\dagger} .
\end{aligned}
$$

¿From (11) and the diagonal character of $e^{-i \Phi}$ we have

$$
l_{n n}=0, \quad v_{m n}=\frac{i l_{m n}}{1-e^{i\left(\varphi_{m}-\varphi_{n}\right)}}, \quad n \neq m .
$$

Differentiating (8) over $\lambda$ we arrive at

$$
\begin{aligned}
& \frac{d \Phi}{d \lambda}=i\left(a-e^{-i \Phi} a e^{i \Phi}\right)+v \\
& \frac{d v}{d \lambda}=[a, v] \\
& \frac{d l}{d \lambda}=[a, l]
\end{aligned}
$$

where

$$
a=\frac{d W}{d \lambda} W^{-1}
$$


For reasons which soon will be clear in the following we shall use the notation:

$$
q_{n}:=\varphi_{n}, \quad p_{n}:=v_{n n},
$$

The diagonal part of the matrix equation (13) reads

$$
\frac{d q_{n}}{d \lambda}=v_{n n}=p_{n}
$$

whereas its off-diagonal part gives the off diagonal elements of $a$ in terms of $v_{m n}$

$$
a_{m n}=\frac{i v_{m n}}{1-e^{-i\left(\varphi_{m}-\varphi_{n}\right)}}, \quad m \neq n
$$

what, upon (12), gives:

$$
a_{m n}=-\frac{l_{m n}}{4 \sin ^{2} \frac{q}{2}}, \quad m \neq n .
$$

By an appropriate choice of the diagonalizing matrix $W$ - see remark, below (8), we can choose $a_{n n}=0$.

Eliminating with the help (12) and (19) $v_{m n}$ and $a_{m n}$ in favour of $l_{m n}$ we obtain from the diagonal part of (14)

$$
\frac{d p_{n}}{d \lambda}=-\sum_{k \neq n} l_{n k} l_{k n} \mathcal{V}^{\prime}\left(q_{k}-q_{n}\right)
$$

and, form (15) for $n \neq m$,

$$
\frac{d l_{m n}}{d \lambda}=-\sum_{k \neq m, n} l_{m k} l_{k n}\left(\mathcal{V}\left(q_{n}-q_{k}\right)-\mathcal{V}\left(q_{k}-q_{m}\right)\right)
$$

where

$$
\mathcal{V}(q)=-\frac{1}{4 \sin ^{2} \frac{q}{2}}
$$

and ' in (21) denotes the derivative with respect to the argument.

Equations (18), (21), and (22) are Hamiltonian with $\lambda$ treated as a fictitious time

$$
\frac{d q_{n}}{d \lambda}=\left\{\mathcal{H}, q_{n}\right\}, \quad \frac{d p_{n}}{d \lambda}=\left\{\mathcal{H}, p_{n}\right\}, \quad \frac{d l_{m n}}{d \lambda}=\left\{\mathcal{H}, l_{m n}\right\}
$$

and with the Hamilton function

$$
\mathcal{H}=\frac{1}{2} \sum_{n=1}^{N} p_{n}^{2}+\frac{1}{2} \sum_{n, m=1}^{N} l_{m n} l_{n m} \mathcal{V}\left(q_{n}-q_{m}\right)=\frac{1}{2} \operatorname{Tr} v^{2}
$$

if we define the following Poisson brackets among the dynamical variables $q_{n}, p_{n}$, and $l_{m n}$

$$
\begin{gathered}
\left\{p_{m}, q_{n}\right\}=\delta_{m n}, \quad\left\{p_{m}, p_{n}\right\}=\left\{q_{m}, q_{n}\right\}=0 \\
\left\{l_{m n}, l_{i j}\right\}=\delta_{i n} l_{m j}-\delta_{m j} l_{i n} \\
\left\{p_{m}, l_{k n}\right\}=\left\{q_{m}, l_{k n}\right\}=0 .
\end{gathered}
$$

It might be appropriate to mention that the system of dynamical equations for the parametric motion of eigenvalues of a Hermitian matrix considered by Yukawa [8] has the same form as (18), (21), and (22) but with a different form of the potential, $\mathcal{V}(q)=-1 / q^{2}$, the 'spatial' coordinates corresponding to eigenvalues, and slightly different definitions of $v$ and $l$. For a unified treatment of parametric motion in autonomous and kicked cases see [14, 20].

The system of equations (18), (21), and (22) can be treated as describing dynamics (in the fictitious time $\lambda$ ) of a one-dimensional gas of particles on the unit circle interacting mutually via the potential (23), but with evolving 'coupling strengths' $l_{m n}$ becoming thus additional dynamical variables. 


\section{STATISTICAL MECHANICS OF THE GAS OF EIGENPHASES}

As the phase-space trajectory of the fictitious gas evolves in time $\lambda$, the original matrix $F(\lambda)$ changes within a oneparameter family. It is that family rather than a single dynamical system (which has a fixed value of $\lambda$ ) which exhibits random-matrix type spectral fluctuations. Indeed, if the motion is ergodic (we shall show below, that it is indeed, although not on the whole energy surface) implies that $\lambda$ averages of spectral characteristics like the distribution of spacings between adjacent quasienergy levels equal ensemble averages. Of importance is thus the minimal time interval $\Delta \lambda$ needed for time and ensemble averages to become practically equal. Obviously $\Delta \lambda \rightarrow \infty$ is sufficient, but not necessary provision. During the evolution 'particles of the gas' (i.e. in fact, the eigenphases) undergo mutual collisions. Observe that since the potential in (25) is repulsive, they usually do not cross (i.e. do not exchange positions). Such a real crossing of two eigenphases would demand vanishing the respective value of $l_{m n}$. Instead what is usually observed in the region of parameter $\lambda$ corresponding to classically chaotic behaviour, are so called avoided crossings when two neighbouring quasienergies approach a minimal nonzero distance when $\lambda$ changes. In fact, as numerical experiments show, for systems which are classically chaotic such avoided crossings are abundant [13]. Due to collisions the gas reaches state in which the motion of particles consists of fluctuations in the vicinity of equilibrium. If $H_{0}$ is integrable and chaos develops gradually after switching the perturbation $V$ and increasing the coupling strength $\lambda$, the spectrum of $F(\lambda)$ equilibrate to RMT predictions only after certain 'relaxation time' when the phase space regions of regular motion have shrunk to relatively negligible weight. If, on the other hand, as shown in [13], $H_{0}$ and $V$ are both non-integrable the initial state of the fictitious gas is already close to equilibrium, and then the window $\Delta \lambda$ in question need not be much larger than the collision time $\lambda_{\text {coll }}$ of the gas, i.e. the mean distance of avoided crossings for a pair of neighbouring levels. It was shown in [13] that the time elapsing between consecutive collisions scales as $N^{-\nu}, \nu>0$. A $\lambda$-average for the over a window $\Delta \lambda \propto \lambda_{\text {coll }}$ thus involves a family of operators $F(\lambda)$ which all yield identical classical dynamics in the limit $N^{-\nu} \rightarrow 0$.

The most straightforward application of statistical mechanics is to employ the canonical ensemble for the distribution of the dynamical variables $(q, p, l)$

$$
\rho(q, p, l) \propto \exp (-\beta \mathcal{H}(q, p, l))
$$

A straightforward integration over Gauss-distributed $p$ and $l$ gives precisely the eigenphase density of random-matrix theory [2], i.e.

$$
P\left(q_{1}, \ldots, q_{N}\right)=\int d^{N} p d^{N(N-1) / 2} l \mathrm{e}^{-\beta \mathcal{H}} \propto \prod_{m<n}\left|\mathrm{e}^{-\mathrm{i} q_{m}}-\mathrm{e}^{-\mathrm{i} q_{n}}\right|
$$

As explained in the introduction the reasoning would be reasonable, if there were no other integrals of motion beside $\mathcal{H}$ itself. In the case other integrals of motion $I_{\mu}$ exist, the appropriate ensemble to use is the generalized canonical ensemble (7).

\section{INTEGRALS OF MOTION}

The equations (18), (21), and (22) are clearly integrable (they can be solved simply by diagonalizing $F$ at given $\lambda$ and calculating appropriate matrix elements), so one should expect that there are much more integrals of motion than the Hamilton function (25) itself. Indeed from (13) and (14) we see that the quantities

$$
I_{k_{1} m_{1} \ldots k_{n} m_{n}}=\operatorname{Tr}\left(v^{k_{1}} l^{m_{1}} \cdots v^{k_{n}} l^{m_{n}}\right) \text {, }
$$

are indeed constants of motion, i.e.

$$
\frac{d}{d \lambda} I_{k_{1} m_{1} \ldots k_{n} m_{n}}=0
$$

and should be taken into account when constructing the generalized canonical ensemble (7). It can be shown [3, 17, 18], that such an ensemble yields the distribution of level spacings as well as low-order correlation functions of the level density in common with random-matrix theory, to within corrections of order $1 / N$. The only problem is whether all integrals nailing down an invariant manifold are of the form (31).

It is possible to show that only $N^{2}-N$ of such integrals are independent. Indeed, let us briefly recall the reasoning presented in [19] and instead of $I_{k_{1} m_{1} \ldots k_{n} m_{n}}$ consider $N^{2}$ quantities

$$
C_{k m}:=\operatorname{Tr}\left(e^{i \Phi} v^{k} e^{-i \Phi} v^{m}\right), \quad 0 \leq k, m \leq N-1 .
$$


¿From the definition of $l$ (11)

$$
e^{i \Phi} v e^{-i \Phi}=v-i l
$$

i.e.

$$
e^{i \Phi} v^{k} e^{-i \Phi} v^{m}=(v-i l)^{k} v^{m}
$$

hence $C_{k m}$ are linear combinations of (31).

1. Not all $C_{k m}$ are independent. Indeed, trivially $C_{k 0}=C_{0 k}=\operatorname{Tr} V^{k}$.

2. There are no more independent integrals of this type (i.e. with $k \geq N$ or $m \geq N$ ). Indeed from the CayleyHamilton theorem applied to the matrix $v$, a $C_{k m}$ with $k$ or $m$ larger than $N-1$ can be expressed a linear combination of the basic ones (33).

Using (8), and (10) we can rewrite $C_{k m}$ as

$$
\begin{aligned}
C_{k m} & =\operatorname{Tr}\left(W F^{\dagger} W^{\dagger} v^{k} W F W^{\dagger} v^{m}\right) \\
& =\operatorname{Tr}\left(F^{\dagger}\left(W^{\dagger} v^{k} W\right) F\left(W^{\dagger} v^{m} W\right)\right) \\
& =\operatorname{Tr}\left(F^{\dagger} V^{k} F V^{m}\right) \\
& =\operatorname{Tr}\left(e^{-i \lambda V} U_{0} V^{k} U_{0}^{\dagger} e^{i \lambda V} V^{m}\right) \\
& =\operatorname{Tr}\left(U_{0} V^{k} U_{0}^{\dagger} V^{m}\right)
\end{aligned}
$$

In the basis in which $V$ is diagonal i.e. $V_{i j}=V_{i} \delta_{i j}$ this reduces to

$$
C_{k m}=\sum_{p, q=1}^{N}\left(V_{q}\right)^{k}\left(V_{p}\right)^{m}\left|\left(U_{0}\right)_{p q}\right|^{2}, \quad 0 \leq k, m \leq N-1 .
$$

Now (33) can be treated as a system of $N^{2}$ linear equations for $N^{2}$ unknown quantities $\left|\left(U_{0}\right)_{p q}\right|^{2}, 1 \leq p, q \leq N$, to be expressed in terms of $N^{2}$ quantities $C_{k m}, 0 \leq k, m \leq N-1$. For a generic $V$ i.e. when all its eigenvalues $V_{i}$ are different the system can be solved, since the determinant of the coefficient matrix is a power of the Vandermonde determinant $D_{V}$ constructed from $V_{i}$,

$$
D_{V}:=\left|\begin{array}{cccc}
1 & V_{1} & \ldots & V_{1}^{N-1} \\
1 & V_{2} & \ldots & V_{2}^{N-1} \\
\vdots & \vdots & \ddots & \vdots \\
1 & V_{N} & \ldots & V_{N}^{N-1}
\end{array}\right|=\prod_{i<j}\left(V_{i}-V_{j}\right)
$$

Thus all $C_{k m}$ can be expressed as linear combinations of $\left|\left(U_{0}\right)_{p q}\right|^{2}$. The $N^{2}$ real numbers $u_{i j}:=\left|\left(U_{0}\right)_{p q}\right|$ fulfill $2 N$ relations stemming from the normalization of rows:

$$
\sum_{j=1}^{N} u_{i j}^{2}=1, \quad i=1, \ldots, N
$$

and columns

$$
\sum_{i=1}^{N} u_{i j}^{2}=1, \quad j=1, \ldots, N
$$

of the unitary matrix $U_{0}$. The number of independent relations is equal to $2 N-1$ and is less by one than the total number of equations in (39) and (40) since summing all equations in (39) over $i$ gives the same as summing all equations in (40) over $j$, namely $N=\operatorname{Tr} U_{0} U_{0}^{\dagger}$. Finally thus all $\left|\left(U_{0}\right)_{p q}\right|^{2}$ involve $N^{2}-(2 N-1)=(N-1)^{2}$ independent parameters and the number of independent $C_{k m}$ which can be used to determine them must be at least equal. Since $C_{k 0}$ and $C_{0 k}$ do not depend at all on $U_{0}$, they can not be used to determine $\left|\left(U_{0}\right)_{p q}\right|^{2}$. To do this we are left only with $C_{k m}, 1 \leq k, m \leq N$ which are exactly $(N-1)^{2}$ in number. The $U_{0}$ - independent integrals $C_{k 0}, 0 \leq k \leq N$, on the other hand, can be used to determine $N$ independent parameters of $V$ (traces of its powers, or what is equivalent, 
its eigenvalues), so they are also independent. Since, trivially, $C_{00}=N$, we are left with the independent integrals of motion of the form:

$$
C_{k}:=C_{k 0}=\operatorname{Tr} V^{k}, \quad k=1,2, \ldots, N-1
$$

which are $N-1$ in number, and $(N-1)^{2}$ integrals

$$
C_{k m}=\operatorname{Tr}\left(U_{0} V^{k} U_{0}^{\dagger} V^{m}\right), \quad k, m=1,2, \ldots, N-1,
$$

i.e. all together $N^{2}-N$ independent integrals of motion.

Let us now count how many variables we have in (18), (21), and (22). The variables $q_{n}$ are real as eigenphases of the unitary matrix $F$ and there are $N$ of them. Also $p_{n}$ as diagonal elements of a Hermitian matrix $v$ are real, there are $N$ of them as well. Since $l$ is antihermitian and off-diagonal there are $\left(N^{2}-N\right) / 2$ matrix elements $l_{m n}$, but since they are, in the case of a general unitary matrix, complex, we should count separately their real and imaginary parts. Finally thus we have $N+N+\left(N^{2}-N\right)=N^{2}+N$ real variables. Comparing this with the number of found integrals of motion $\left(N^{2}-N\right)$ we are tempted to think that, in a generic case, invariant manifolds are of the dimension $\left(N^{2}+N\right)-\left(N^{2}-N\right)=2 N$.

On the other hand, in the coordinate frame in which $V$ is diagonal, $V_{i j}=V_{i} \delta_{i j}$, the motion described by $F(\lambda)=$ $\exp (-i V) U_{0}$ involves only $N$ independent frequencies $V_{k}$, and takes place on an $N$-dimensional torus (and is ergodic on it i a generic case when the eigenvalues of $V$ are not rationally dependent). It seems thus, we are still missing $N$ independent integrals of motion.

\section{POISSON STRUCTURE AND CONSTRAINTS}

Before identifying missing integrals and determining their influence (or lack of) on the distribution of eigenphases, let's consider more carefully the proposed Hamiltonian formulation. First, observe that the definition of the manifold on which the level dynamics takes place as parameterized by the coordinates $q_{n}, p_{n}$ and $l_{m n}$ and equipped with the Poisson structure (26)-(28) is slightly flawed. From the definition (11) of $l$ we have $l_{n n}=0$, but this is inconsistent with the Jacobi identity which must be fulfilled by (28):

$$
\left\{l_{p q},\left\{l_{i k}, l_{m n}\right\}\right\}+\left\{l_{i k},\left\{l_{m n}, l_{p q}\right\}\right\}+\left\{l_{m n},\left\{l_{p q}, l_{i k}\right\}\right\}=0 \text {. }
$$

Indeed, substituting to the above $m=k$ and $n=i$ and using $l_{k k}=l_{i i}=0$ whenever they appear on intermediate steps, we arrive at an erroneous result $\delta_{p q} l_{k q}+\delta_{i q} l_{p i}-\delta_{k q} l_{p k}-\delta_{p i} l_{i q}$. instead of zero. Thus we are not allowed to put $l_{n n}=0$ from the very beginning as equations defining our manifold. Instead, if we want to keep the Poisson brackets (28) we should change the definition (11) to

$$
l=i e^{i \Phi}\left[v, e^{-i \Phi}\right]+i L
$$

where $L$ is an arbitrary, real, diagonal matrix, i.e. we introduced $N$ additional dynamical variables. To understand their meaning let us return to the derivation of the dynamical equations by diagonalizing matrix $W$ (8), but this time we do not impose additional conditions on $W$, i.e. we do not assume that the diagonal matrix elements $a_{n n}$ of $a=d W / d \lambda \cdot W^{-1}$ vanish. Instead we allow them to be arbitrary functions of $\lambda$. It should be clear (and indeed we will show that it is the case), nothing really depends on the choice of $a_{n n}$, since nothing concerning the eigenvalues should depend on the choice of the diagonalizing matrix.

The resulting equations of motion are derived in the same way as previous ones (18), (21), and (22). In fact only the third of them is altered and reads now,

$$
\begin{aligned}
\frac{d l_{m n}}{d \lambda}= & -\sum_{k \neq m, n} l_{m k} l_{k n}\left(\mathcal{V}\left(q_{n}-q_{k}\right)-\mathcal{V}\left(q_{k}-q_{m}\right)\right) \\
& +l_{m n}\left(a_{m m}-a_{n n}\right)+l_{m n}\left(l_{n n}-l_{m m}\right) \mathcal{V}\left(q_{m}-q_{n}\right)
\end{aligned}
$$

Equations (18), (21), (45) are again Hamiltonian with the same Poisson structure (26)-(28), but with a new Hamilton function

$$
\mathcal{H}=\frac{1}{2} \sum_{n=1}^{N} p_{n}^{2}+\frac{1}{2} \sum_{n, m=1}^{N} l_{m n} l_{n m} \mathcal{V}\left(q_{n}-q_{m}\right)+\sum_{j}^{N} a_{j j} l_{j j}
$$


depending on $N$ arbitrary (in general 'time-', i.e. $\lambda$-dependent) functions $a_{n n}$. The quantities $C_{m n}$ (33) are again integrals of motion. In addition, we easily calculate that

$$
\left\{\mathcal{H}, l_{n n}\right\}=0
$$

so $l_{n n}$ are also constants of motion. In fact, as it is clear from the previous considerations, nothing concerning the eigenphases depends on actual values of $l_{n n}$. We can thus impose constraints, eg.

$$
l_{n n}=0, \quad n=1, \ldots, N
$$

At this point it is instructive and in fact very natural to describe the encountered situation from the point of view of Dirac's theory of constrained Hamiltonian systems [21]. The conditions (48) are so called primary constraints (i.e. they are not obtained form the equations of motion) and can be imposed only after evaluating all Poisson brackets to avoid the problems with the Jacobi identity mentioned at the beginning of the present section. Further, one calculates easily that

$$
\left\{l_{m m}, l_{n n}\right\}=0
$$

Together with (47) it means that the consistency condition

$$
\left\{\mathcal{H}, l_{m m}\right\}+\sum_{n=1}^{N} a_{n n}\left\{l_{n n}, l_{m m}\right\}=0
$$

are identically fulfilled and no other constraints, neither primary nor secondary, are produced, nor additional conditions are imposed on the functions $a_{n n}(\lambda)$.

Due to (49) $l_{n n}$ are automatically first-class constraints (recall that according to Dirac terminology a quantity is of first-class if its Poisson brackets with all constraints vanish, see [21], p. 18). The new Hamilton function (46) involves as many arbitrary functions (in our case these are functions $a_{n n}(\lambda)$ ), as there are independent primary firstclass constraints. On the other hand, first-class primary constraints (48) may be always used to produce a gauge transformation generated by

$$
G(\lambda)=\sum_{n=1}^{N} \theta_{n}(\lambda) l_{n n}
$$

i.e.

$$
l_{i j} \mapsto e^{i \theta_{i}(\lambda)} l_{i j} e^{-i \theta_{j}(\lambda)}
$$

with arbitrary $\lambda$-dependent $\theta_{k}, k=1, \ldots, N$.

We expect that an initial physical state determined by initial values of the phase-space variables $\left(q_{n}, p_{n}, l_{m n}\right)$ determines also its all future physical states. Since the Hamilton function (46) depends on $N$ arbitrary functions, the same may happen to the values of $\left(q_{n}, p_{n}, l_{m n}\right)$ at latter times. But the only freedom is now given by the gauge transformation (52) connecting the variables describing the same physical state of the system for different choices of the gauge. Hence particular physical properties of the state (eg. statistical properties of the distribution of positions, ie., in our case, eigenphases) should be gauge-independent, and in fact they are, since the gauge transformation does not influence the relevant variables $q_{n}$.

To be even more concrete in explaining the role of the gauge transformation for the present problem let us observe that by assuming $l_{m m}=0$ we recovered the previous count of the number of variables vs. dimension of the invariant manifold, since the number of variables was first increased by $N$ by introducing the diagonal elements of $l$ and then decreased by the same number by imposing constraints equating them to zero. To fix the (still) remaining $N$ degrees of freedom let us observe that the gauge transformation (52) does not change the integrals of motion (in particular the Hamilton function itself) after reducing to the manifold determined by the constraints (48), retaining also the equations of motion in their original form. The transformation is intimately related to the freedom of choice of the diagonalizing matrix $W$ in terms of $a$, it leads to

$$
a \mapsto i \frac{d \theta}{d \lambda}+e^{i \theta} a e^{-i \theta}, \quad \theta:=\operatorname{diag}\left(\theta_{1}, \ldots, \theta_{2}\right) .
$$

With the help of (52) we can fix in an arbitrary way $N$ (more precisely $N-1$, but one additional is determined by the choice of initial point on the unit circle) phases of the variables $l_{n m}$. Let us summarize 
- number of variables: $N_{v a r}=N^{2}+2 N$ (the old ones plus the (imaginary parts of) diagonal elements of $l$

- number of independent integrals $C_{m n}: N_{i n t}=N^{2}-N$

- number of constraints $l_{n n}=0: N_{c}=N$

- number of phases fixed by choosing a gauge $N_{g}=N$,

hence $N_{v a r}-\left(N_{i n t}+N_{c}+N_{g}\right)=N=$ dimension of the invariant manifold.

Now it is clear that integrals of motion $C_{m n}$ (33) are the only quantities which should be taken into account when determining the equilibrium distribution. Indeed, as already mentioned the constraints and the gauge, involving only $l_{m n}$, do not influence eigenphases, what is a direct consequence of the independence of the eigenvalues on the choice of the diagonalizing matrix. Moreover, our choice $l_{n n}=0$ reduces the Hamilton function (46) to originally considered one (25) and the whole reasoning which led, after integration out of $p$ and $l$ variables and neglecting corrections of order $1 / N$, to random matrix results for the eigenphases, is fully vindicated.

\section{ACKNOWLEDGMENTS}

We enjoyed fruitful discussions with Stefan Giller and Paweł Maślanka. The support by SFB/TR12 Symmetries and Universality in Mesoscopic Systems program of the Deutsche Forschungsgemeischaft and Polish MNiSW grant no. 1P03B04226 is gratefully acknowledged.

[1] O. Bohigas, M. J. Giannoni, and C. Schmit, Phys. Rev. Lett. 52, 1 (1984).

[2] M. L. Mehta, Random Matrices and the Statistical theory of Energy Levels, New York (Academic Press, 1991), 2nd ed.

[3] F. Haake, Quantum Signatures of Chaos (Springer, Berlin, 2000), 2nd ed.

[4] H.-J. Stöckmann, Quantum Chaos: An Introduction (Cambridge University Press, Cambridge, 1999).

[5] S. Müller, S. Heusler, P. Braun, F. Haake, and A. Altland, Phys. Rev. Lett. 93, 014103 (2004).

[6] S. Müller, S. Heusler, P. Braun, F. Haake, and A. Altland, lanl.arXiv.org/nlin.CD/0503052 (2005).

[7] P. Pechukas, Phys. Rev. Lett. 51, 943 (1983).

[8] T. Yukawa, Phys. Rev. Lett. 54, 1883 (1985).

[9] T. Yukawa, Phys. Lett. A 116, 227 (1986).

[10] F. Dyson, J. Math. Phys 3, 140 (1962).

[11] M. Kuś, R. Scharf, and F. Haake, Z. Phys. B 66, 129 (1987).

[12] K. Nakamura and H. J. Mikeska, Phys. Rev. A 35, 5294 (1987).

[13] P. Braun, S. Gnutzmann, F. Haake, M. Kuś, and K. Życzkowski, Found. Phys. 31, 613 (2001).

[14] A. Huckleberry, D. Zaitsev, M. Kuś, and F. Haake, J. Geom. Phys. 37, 156 (2001).

[15] S. Wojciechowski, Phys. Lett. A 111, 101 (1985).

[16] M. Kuś, Europhys. Lett 5, 1 (1988).

[17] B. Dietz and F. Haake, Europhys. Lett. 9, 1 (1989).

[18] B. Dietz, Z. Phys. B 96, 271 (1994).

[19] K. Mnich, Phys. Lett. A 176, 189 (1993).

[20] M. Kuś, F. Haake, D. Zaitsev, and A. Huckleberry, J. Phys. A: Math. Gen. 30, 8635 (1997).

[21] P. A. M. Dirac, Lectures on Quantum Mechanics (Belfer Graduate School of Science, Yeshiva University, New York, 1964). 